\title{
Scoring Neuropsychological Tests: What Corrections Need to be Considered?
}

\author{
Yassar Alamri ${ }^{\mathrm{a}-\mathrm{c}}$ \\ ${ }^{a}$ Christchurch School of Medicine, University of Otago, Christchurch, New Zealand; ${ }^{b}$ Department of Medicine, \\ University of Otago, Christchurch, New Zealand; ' $N e w$ Zealand Brain Research Institute, Christchurch, New Zealand
}

Dear Sir,

We read the findings of Burggraaff et al. [1] with great interest. Several factors seem to affect the participant's performance on a certain neuropsychological test, and hence their score. However, investigating the literature revealed a general paucity of studies accounting for patient demographics (apart from age) and anthropometric factors (e.g., handedness), which may affect the performance. For example, it is not known if a lateralised tremor in Parkinson's disease (PD) patients - regardless of cognitive status - adversely affects their performance on neuropsychological tests.

We take the Brief Visuospatial Memory Test-Revised (BVMT-R) as an example. The BVMT-R is intended to examine visual learning and visuospatial memory. The figures are arranged in a $2 \times 3$ grid on a sheet of paper. The test may be completed via verbal responses (i.e., recall/recognition trials) or written responses (i.e., copy trial). Scoring of the task largely depends on the accurate reproduction of a figure as well as correctly placing it in relation to other figures.

In $\mathrm{PD}$, the BVMT-R has primarily been used as a test of right hemispheric dys- function, given the premise that BVMT-R assesses visuospatial memory. Amick et al. [2] found that PD patients with left-sided symptoms (L-PD) performed significantly worse than $\mathrm{PD}$ patients with right-sided symptoms (R-PD). In another study, Foster et al. administered a battery of neuropsychological tests to a group of patients with R-PD and another with L-PD. Among the tests were the BMVT-R immediate recall and the BVMT-R delayed recall tasks. BVMT-R's scores of patients with R-PD, but not L-PD, significantly improved between the immediate and delayed trials [3].

Whilst the written copy trial may potentially be negatively affected by conditions that result in motor dysfunction (e.g., PD and stroke), verbal recall/recognition trials may be affected by motor slowing in more subtle ways. For example, patients with schizophrenia have been shown to exhibit "inefficient" oculomotor behaviour on the Symbol-Digit Modalities Test leading to significantly lower scores compared with controls, independent of overt slowing in axial or appendicular musculature [4]. Therefore, excursions may be warranted to accurately reflect the underlying neurocognitive impairment regardless of the patient's behavioural choices (e.g., smoking status; 5 or mechanical impairment).

Additional non-demographicallybased corrective factors may be required for certain patient populations completing various neuropsychological assessments. Whether such corrections would result in better clinical characterisation of these patients, however, remains to be answered. A longitudinal study is being currently undertaken on the performance of PD patients on the BVMT-R (Alamri et al., unpublished). Whilst such study focusses on PD patients, it would be imperative to take caution when applying "normative data" to particular patient groups, especially if specific corrections (e.g., sex, education level or motor impairment) need to be applied.

\section{Disclosure Statement}

The author has no conflict of interest to declare.

\section{KARGER}

(c) 2017 S. Karger AG, Basel
Yassar Alamri, $\mathrm{MBChB}, \mathrm{PhD}$

New Zealand Brain Research Institute 66 Stewart Street

Central Christchurch 8011 (New Zealand)

E-Mail yassar.alamri@nzbri.org 


\section{References}

1 Burggraaff J, Knol DL, Uitdehaag BMJ: Regression-based norms for the symbol digit modalities test in the dutch population: improving detection of cognitive impairment in multiple sclerosis? Eur Neurol 2017;77:246252
2 Amick MM, Grace J, Chou KL: Body side of motor symptom onset in Parkinson's disease is associated with memory performance. J Int Neuropsychol Soc 2006;12:736-740.

3 Foster PS, et al: Verbal and visuospatial memory in lateral onset Parkinson disease: time is of the essence. Cogn Behav Neurol 2010; 23:19-25.
4 Elahipanah A, Christensen BK, Reingold EM: What can eye movements tell us about symbol digit substitution by patients with schizophrenia? Schizophr Res 2011;127:137-143.

5 Reed AC, Harris JG, Olincy A: Schizophrenia, smoking status, and performance on the matrics cognitive consensus battery. Psychiatry Res 2016;246:1-8. 